\title{
Diagnostic likelihood ratio
}

Margaret Pepe

\section{Source}

Margaret Sullivan Pepe. (2003). The Statistical Evaluation of Medical Tests for

Classification and Prediction Oxford University Press

Diagnostic likelihood ratios are the ratios of the likelihood of the observed test result in diseased vs. non-diseased subjects. The positive diagnostic likelihood ratio (DLR+) is the ratio between the true positive fraction and the false positive fraction. The negative diagnostic likelihood ratio (DLR-) is the ratio between the false negative fraction (i.e. 1 true positive fraction) and the true negative fraction (i.e. 1 - false positive fraction). A DLR+ $>1$ indicates that a positive test is more likely in a diseased than in a non-diseased subject. A DLR $-<1$ indicates that a negative test is more likely in a diseased than in a non-diseased subject. 